\title{
Stability Properties of Adams-Moulton Type Methods
}

\author{
By Robert R. Brown, James D. Riley and Morris M. Bennett
}

I. Introduction. A recent study by Chase [1] was directed toward the stability of certain predictor-corrector methods. In particular, he studied an iterated Milne, a predictor-corrector Milne, an iterated Hamming, and a predictor-corrector Hamming.

A recent paper by Feldstein and Stetter [2] suggests a one-derivative evaluation per step variation of the Adams-Moulton procedure. Hull and Creemer [3] suggest a two-correction, two-derivative evaluation per step method.

These two methods plus the classical Adams-Moulton, both iterative and predictor-corrector, are studied. Also two variations on the above that subtract out local truncation error are included. These variations convert the fourth-order procedure to fifth order with no further derivative evaluations.

1 The methods used, as well as the notation, are similar to Chase [1]. In the rootlocus graphs, magnitudes only are plotted. A square is a negative root, a triangle a pair of complex conjugates, and a circle a positive root.

Obvious extensions of these results could be considered. One extension would be to consider $p$ corrections and derivative evaluations for arbitrary $p$. However, few programs consider $p>2$ without iterating to approximate convergence. Another extension would be to consider Adams-Moulton methods of arbitrary order. However, the majority of AM programs use the fourth-order procedure and the extension would involve considerable effort. Therefore, these extensions, while certainly of theoretical interest, will not be considered at this time.

\section{Methods Considered.}

Method A (Feldstein-Stetter)

$$
\begin{aligned}
& x_{n+1}=y_{n}+\frac{h}{24}\left(55 x_{n}^{\prime}-59 x_{n-1}^{\prime}+37 x_{n-2}^{\prime}-9 x_{n-3}^{\prime}\right), \\
& y_{n+1}=y_{n}+\frac{h}{24}\left(9 x_{n+1}^{\prime}+19 x_{n}^{\prime}-5 x_{n-1}^{\prime}+x_{n-2}^{\prime}\right) .
\end{aligned}
$$

The characteristic equation is

$$
\rho^{5}-\left(\frac{8 \hbar}{3}+1\right) \rho^{4}+\frac{95 \bar{h}}{24} \rho^{3}-\frac{91 \hbar}{24} \rho^{2}+\frac{15 \bar{h}}{8} \rho-\frac{3 \hbar}{8}=0 .
$$

The root locus is shown in Figure 1. Note that -1 is a root when $\bar{h}=-\frac{3}{18}$.

Method B (Adams-Moulton)

$$
x_{n+1}=y_{n}+\frac{h}{24}\left(55 y_{n}^{\prime}-59 y_{n-1}^{\prime}+37 y_{n-2}^{\prime}-9 y_{n-3}^{\prime}\right),
$$

Received January 6, 1964. 


$$
y_{n+1}=y_{n}+\frac{h}{24}\left(x_{n+1}^{\prime}+19 y_{n}^{\prime}-5 y_{n-1}^{\prime}+y_{n-2}^{\prime}\right) .
$$

The characteristic equation is

$$
\rho^{4}-\left(\frac{55 \bar{h}^{2}}{64}+\frac{7 \bar{h}}{6}+1\right) \rho^{3}+\left(\frac{59 \bar{h}^{2}}{64}+\frac{5 \bar{h}}{24}\right) \rho^{2}-\left(\frac{37 \bar{h}^{2}}{64}+\frac{h}{24}\right) \rho+\frac{9 \bar{h}^{2}}{64}=0 .
$$

The root locus is shown in Figure 2 .

Method C (Adams-Moulton with second correction)

$$
\begin{aligned}
& x_{n+1}=z_{n}+\frac{h}{24}\left(55 y_{n}^{\prime}-59 y_{n-1}^{\prime}+37 y_{n-2}^{\prime}-9 y_{n-3}^{\prime}\right), \\
& y_{n+1}=z_{n}+\frac{h}{24}\left(9 x_{n+1}^{\prime}+19 y_{n}^{\prime}-5 y_{n-1}^{\prime}+y_{n-2}^{\prime}\right), \\
& z_{n+1}=z_{n}+\frac{h}{24}\left(9 y_{n+1}^{\prime}+19 y_{n}^{\prime}-5 y_{n-1}^{\prime}+y_{n-2}^{\prime}\right) .
\end{aligned}
$$

The characteristic equation is

$$
\rho^{5}-\left(\bar{h}^{2}+\frac{7 \bar{h}}{6}+1\right) \rho^{4}+\left(\frac{95 \bar{h}^{2}}{64}+\frac{5 \bar{h}}{24}\right) \rho^{3}-\left(\frac{91 \bar{h}^{2}}{64}+\frac{\bar{h}}{24}\right) \rho^{2}+\frac{45 \bar{h}^{2}}{64} \rho-\frac{9 \bar{h}^{2}}{64}=0 .
$$

The root locus is shown in Figure 3.

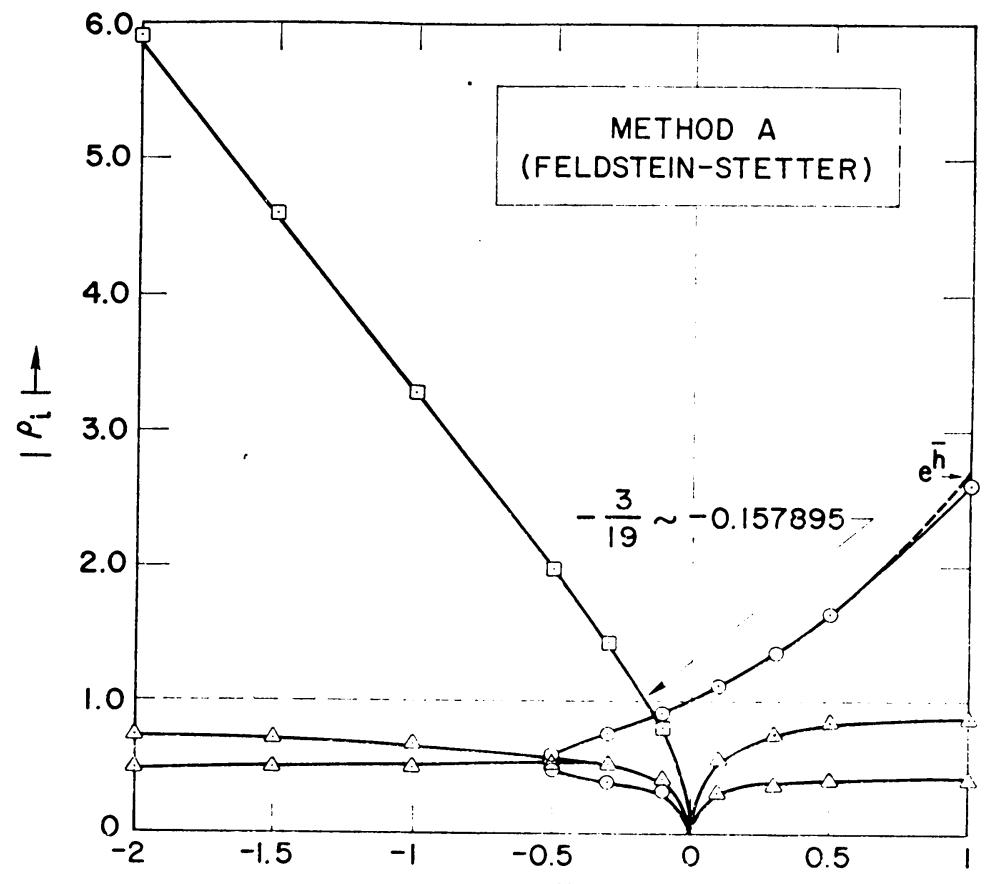

$(\bar{h})$

FIG. 1 


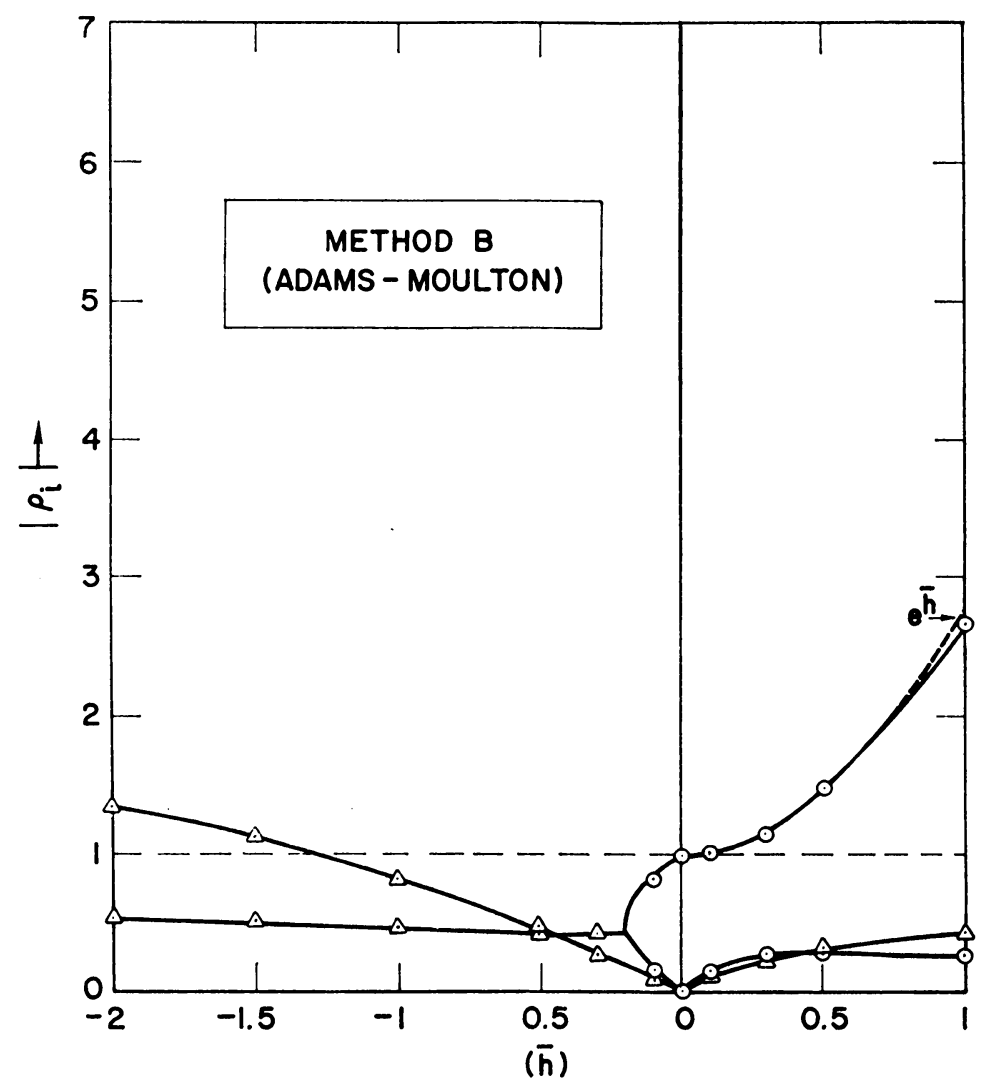

Fig. 2

\section{Method D (Iterated Adams-Moulton)}

If the Adams-Moulton corrector is iterated to convergence, the characteristic equation is

$$
\left(1-\frac{3 \hat{h}}{8}\right) \rho^{3}-\left(1+\frac{19 \bar{h}}{24}\right) \rho^{2}+\frac{5 \bar{h}}{24} \rho-\frac{\bar{h}}{24}=0 .
$$

The root locus is shown in Figure 4. Note that setting $\rho=-1$ gives $\bar{h}=-3$. The positive root is about 10.17 when $\bar{h}=2$.

Method E (Adams-Moulton with second correction with estimated local truncation error removed)

$$
\begin{aligned}
& x_{n+1}=w_{n}+\frac{h}{24}\left(55 u_{n}^{\prime}-59 u_{n-1}^{\prime}+37 u_{n-2}^{\prime}-9 u_{n-3}^{\prime}\right), \\
& y_{n+1}=x_{n+1}-\frac{251}{270}\left(x_{n}-v_{n}\right), \\
& z_{n+1}=w_{n}+\frac{h}{24}\left(9 y_{n+1}^{\prime}+19 u_{n}^{\prime}-5 u_{n-1}^{\prime}+u_{n-2}^{\prime}\right), \\
& u_{n+1}=z_{n+1}+\frac{19}{270}\left(x_{n+1}-z_{n+1}\right),
\end{aligned}
$$




$$
\begin{aligned}
v_{n+1} & =w_{n}+\frac{h}{24}\left(9 u_{n+1}^{\prime}+19 u_{n}^{\prime}-5 u_{n-1}^{\prime}+u_{n-2}^{\prime}\right)=z_{n+1}+\frac{3 h}{8}\left(u_{n+1}^{\prime}-y_{n+1}^{\prime}\right), \\
w_{n+1} & =v_{n+1}+\frac{19}{270}\left(x_{n+1}-v_{n+1}\right) .
\end{aligned}
$$

The characteristic equation is given by
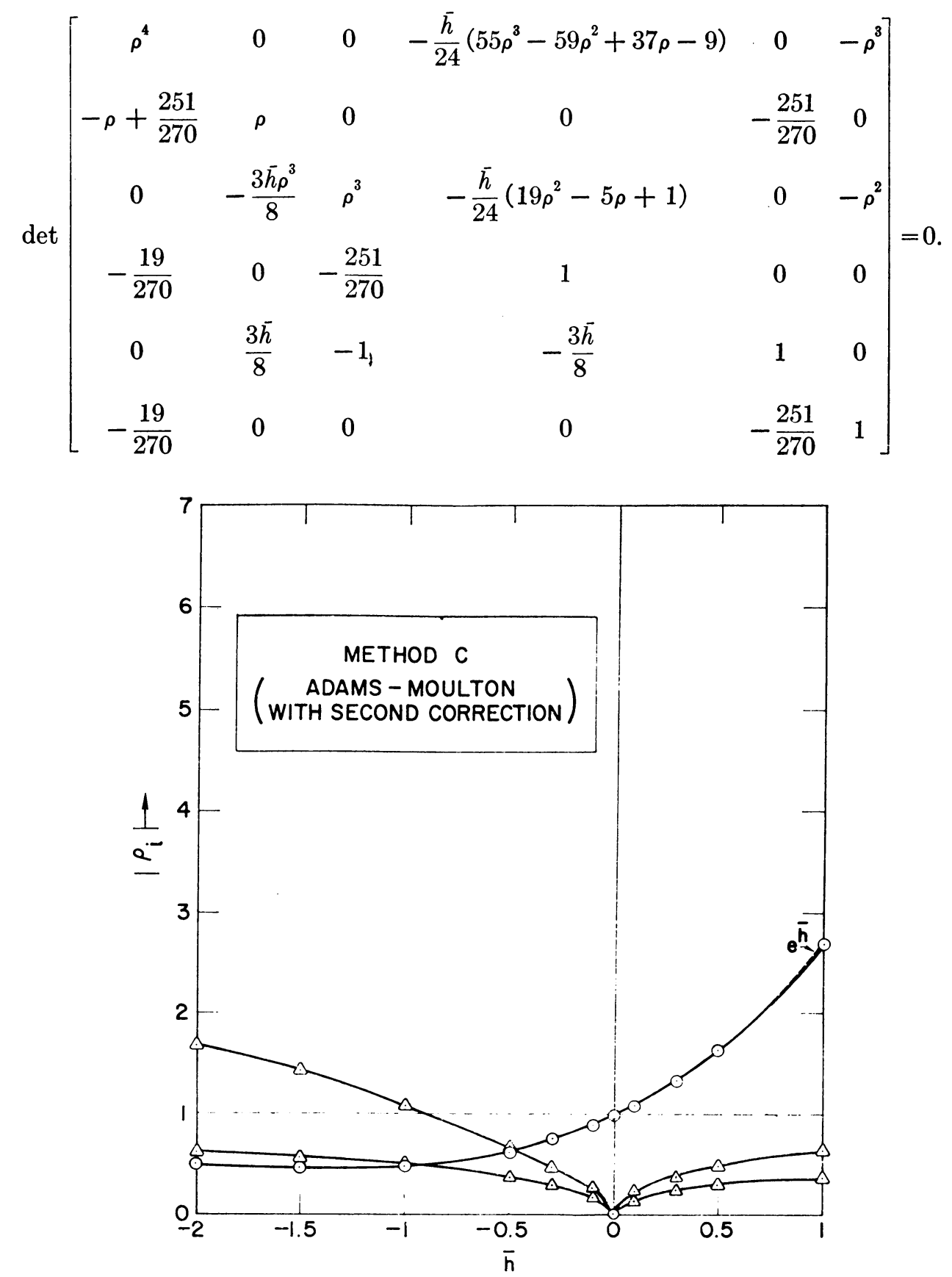

FIG. 3 


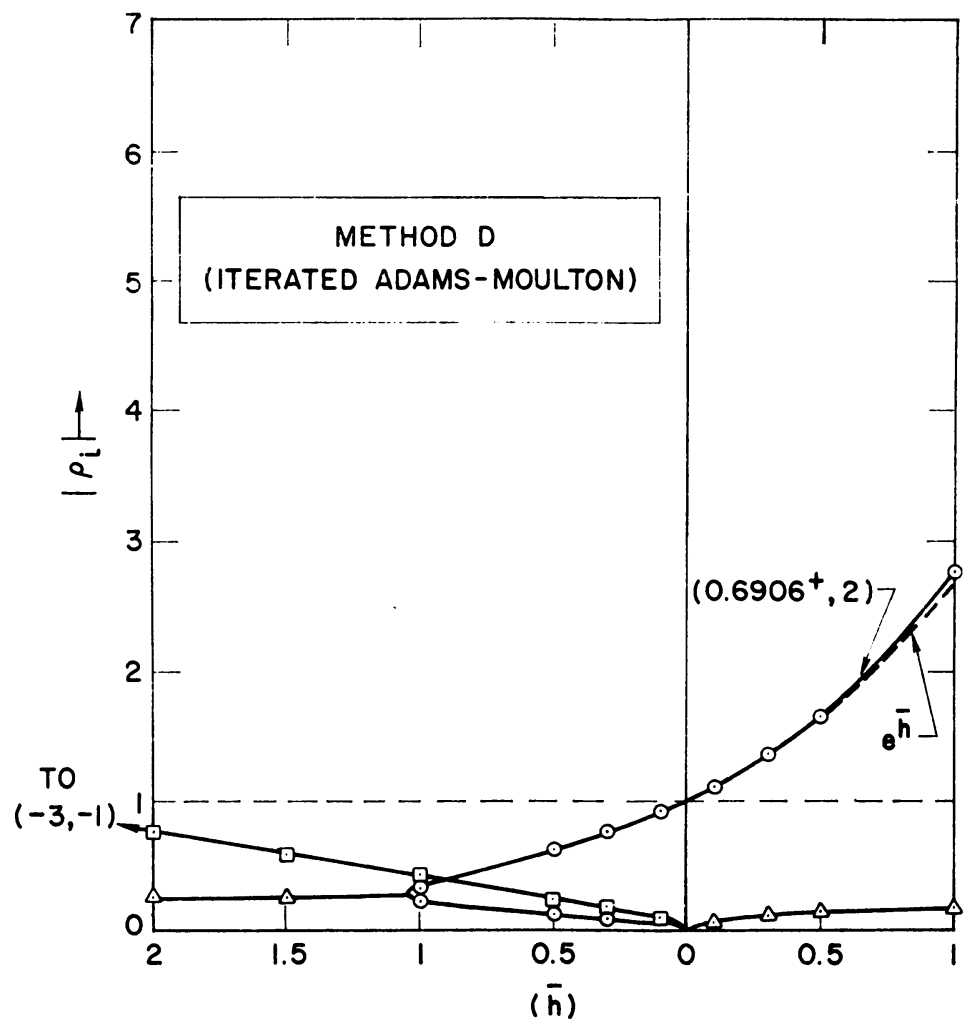

Fig. 4

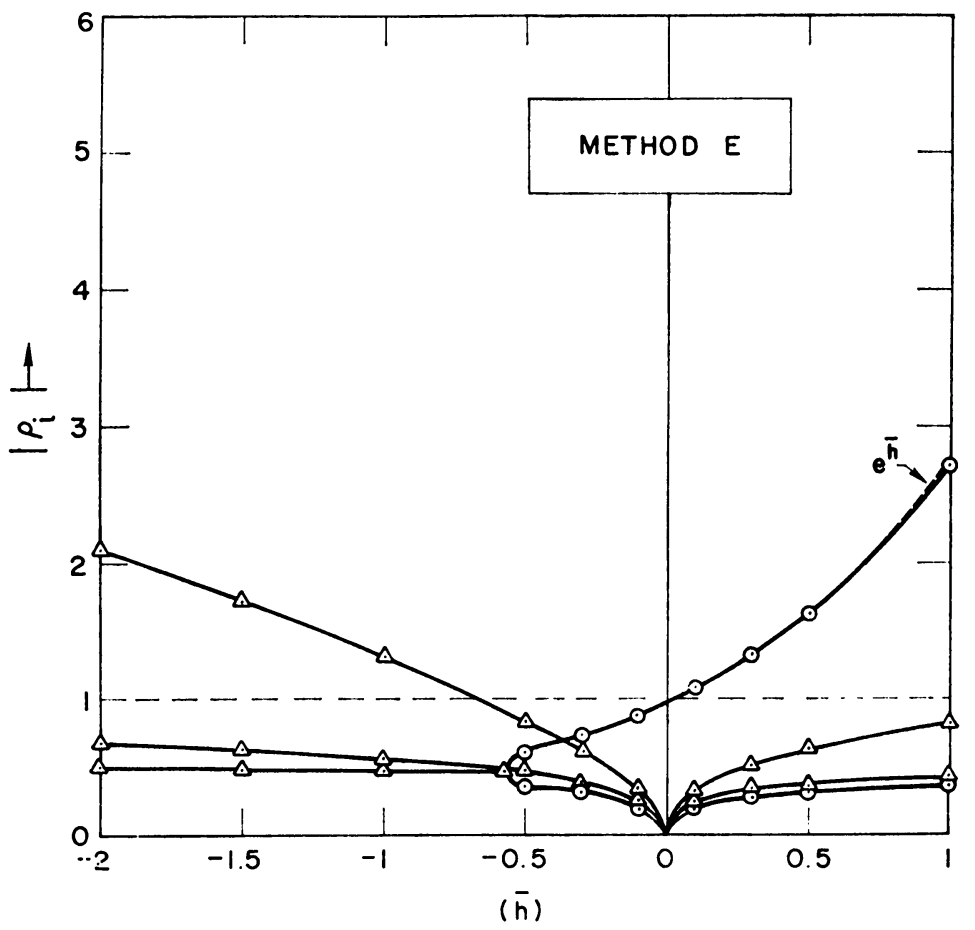

FIg. 5 


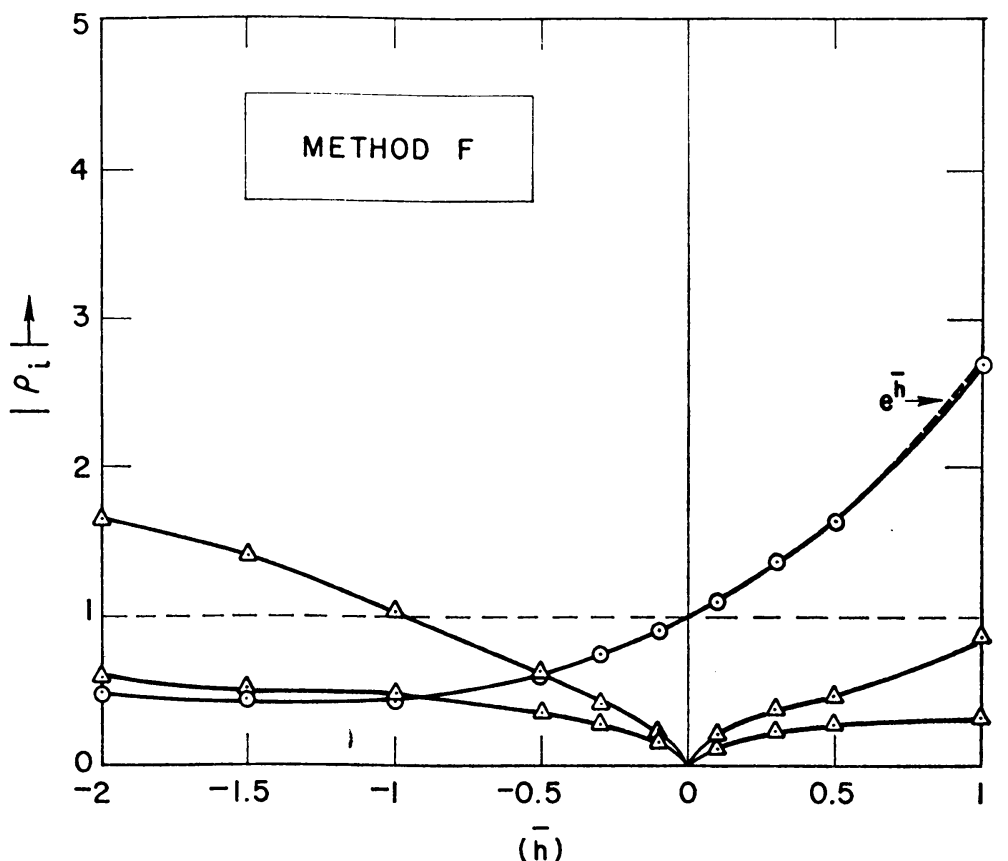

FIg. 6

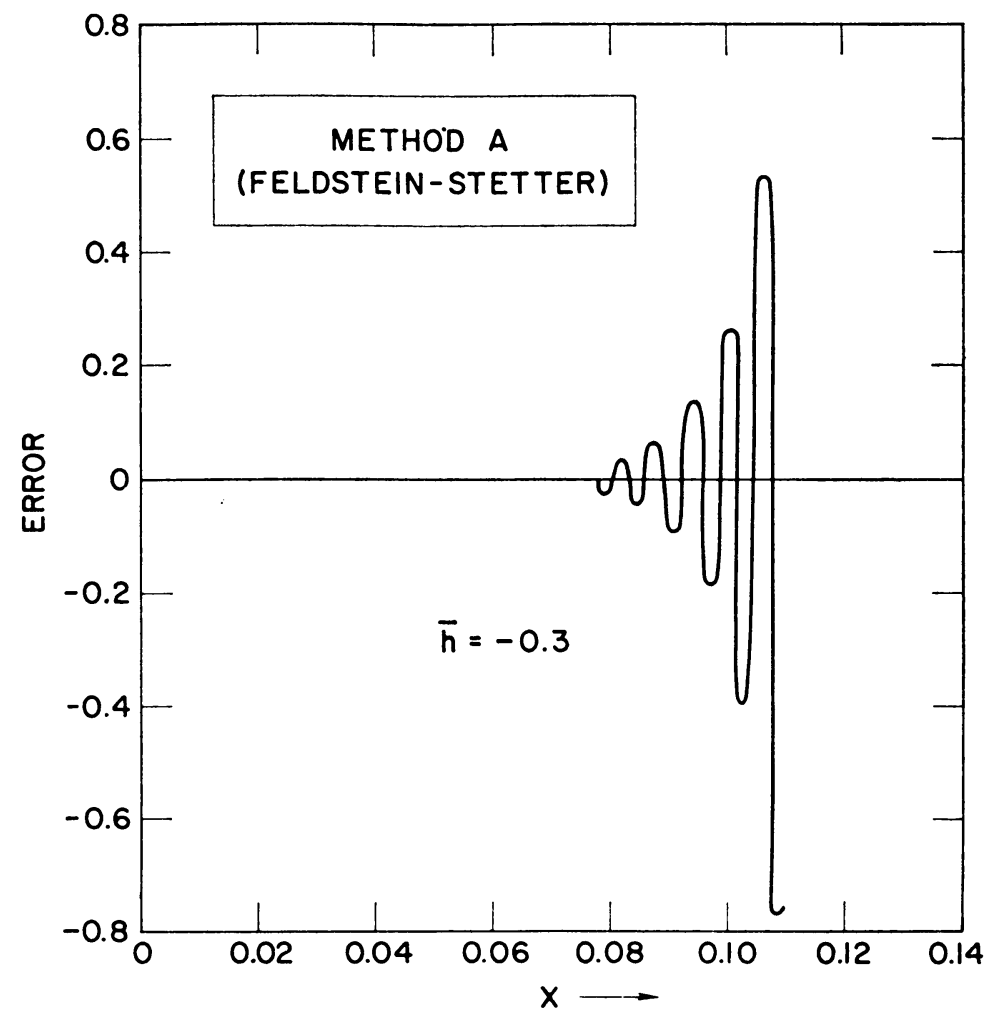

FIG. 7 
The root locus is shown in Figure 5 (after eliminating a factor of $\rho^{2}$ ).

\section{Method F}

A variation of Method $E$ skips the attempt to remove the local truncation error in the predictor formula. The characteristic determinant is obtained from the one above by adding the second column to the first column and then removing the second row and column to form a fifth-order determinant. The root locus is shown in Figure 6.

Note that the variation improves the stability.

III. Error Curves. The differential equation is $y^{\prime}=-100 y+100$ with $y(0)=0$, which has the solution $y=1-e^{-100 x}$. This makes $\bar{h}=-100 h$.

In Figure 7 is the error curve for Method A (Feldstein-Stetter) with $\bar{h}=-.3$. At $x=.078$, the error is -.0229206 . At $x=.108$, ten steps later, the error is -.772704 . Solving for $\rho$ in the equation $(-\rho)^{10}=.772704 \div .0229206$ gives $\rho=-1.4216$. The characteristic polynomial with $\bar{h}=-.3$ was found to have a root of -1.4216 .

Similar checks have been made for Methods B, C, E, and F. Methods E and F were found to be substantially more accurate in the stable range, as would be expected from their higher order.

IV. Conclusions. Method A has a very small stability range for negative $\bar{h}$ and therefore should be used with caution.

The extra application of the corrector in $\mathrm{C}$ gives poorer stability than $\mathrm{B}$ and could be harmful if it caused instability.

Method F has better stability than Method E, and appears to have no disadvantages in comparison to Method E. It is also simpler than E.

Aerospace Corporation

El Segundo, California

1. P. E. ChASE, "Stability properties of predictor-corrector methods for ordinary differential equations," J. Assoc. Comput. Mach., v. 9, 1962, p. 457-468. MR 29 *738.

2. M. A. Feldstein \& H. J. STeTter, Simplified Predictor-Corrector Methods, ACM National Conference, August 27, 1963.

3. J. E. Hull \& A. L. CREemer, "Efficiency of predictor-corrector procedures," J. As8oc. Comput. Mach., v. 10, 1963, p. 291-301. MR $27 * 4367$.

4. R. W. HAMMING, "Stable predictor-corrector methods for ordinary differential equations," J. Assoc. Comput. Mach., v. 6, 1959, p. 37-47. MR $21 * 973$. 